\title{
Redox cycling in nanofluidic channels using interdigitated electrodes
}

\author{
Edgar D. Goluch • Bernhard Wolfrum • \\ Pradyumna S. Singh • Marcel A. G. Zevenbergen • \\ Serge G. Lemay
}

Received: 10 September 2008 /Revised: 1 December 2008 / Accepted: 8 December 2008 / Published online: 6 January 2009

(C) Springer-Verlag 2008

\begin{abstract}
Amperometric detection is ideally suited for integration into micro- and nanofluidic systems as it directly yields an electrical signal and does not necessitate optical components. However, the range of systems to which it can be applied is constrained by the limited sensitivity and specificity of the method. These limitations can be partially alleviated through the use of redox cycling, in which multiple electrodes are employed to repeatedly reduce and oxidize analyte molecules and thereby amplify the detected signal. We have developed an interdigitated electrode device that is encased in a nanofluidic channel to provide a hundred-fold amplification of the amperometric signal from paracetamol. Due to the nanochannel design, the sensor is resistant to interference from molecules undergoing irreversible redox reactions. We demonstrate this selectivity by detecting paracetamol in the presence of excess ascorbic acid.
\end{abstract}

Keywords Nanotechnology · Nanofluidic ·

Electrochemistry · Amperometric detection · Redox cycling . Interdigitated electrodes (IDEs) · Paracetamol .

Ascorbic acid $\cdot$ Sensor

E. D. Goluch • B. Wolfrum • P. S. Singh • M. A. G. Zevenbergen •

S. G. Lemay $(\bowtie)$

Kavli Institute of Nanoscience, Delft University of Technology, Lorentzweg 1, 2628 CJ Delft, The Netherlands

e-mail: S.G.Lemay@tudelft.nl

Present address:

B. Wolfrum

IBN-2, Forschungszentrum Jülich $\mathrm{GmbH}$,

JARA-FIT,Wilhelm-Johnen Straße,

52428 Jülich, Germany

\section{Introduction}

Amperometric detection, in which the faradaic current generated by redox reactions at an electrode is used to determine the composition of a sample, is in principle an ideal detection method for integration into nanofluidic analysis systems. First, it directly provides an electrical signal that is proportional to the concentration of a redoxactive analyte. Low-cost, robust devices can thus be fabricated without the need to integrate cumbersome optical components. Second, real-time, label-free detection in nanochannels is especially difficult as most analytical tools currently lack the necessary sensitivity at these volumes.

Amperometric detection however has limitations that have so far hindered its broad deployment. Most importantly, it suffers from limited specificity: typical samples of interest in medical or environmental monitoring applications contain multiple chemical species that react at similar electrode potentials, rendering it difficult or impossible to discriminate between these different species. Many different techniques have been developed to enhance selectivity. These include for example sample pretreatment by enzymatic reactions [1, 2], chemical surface modification [3-6], and sample pre-separation by electrophoresis [7-9]. These approaches can only be applied in very specific situations, however, and further progress must occur before amperometric detection can become more widely applicable in labon-a-chip technology.

Recent years have seen important progress in the ability to control and fashion matter on the nanometer scale, and the field of electrochemistry is no exception. The extension of amperometric detection methods to the nanoscale has already yielded much new information about mass transfer [10-12], adsorption [13, 14], and 
electron-transfer kinetics [15-17] that would be otherwise difficult to observe. One goal of nanofluidics, the handling and probing of individual molecules, is already becoming a reality $[16,18]$. As a further example, miniature probes are increasingly employed for in vivo measurements of neurotransmitters in the brain [19-23]. Despite these successes, however, the use of electrochemistry in nanofluidics remains in its infancy.

In this paper, we focus on one particular approach in amperometric detection: redox cycling. This approach employs multiple electrodes to reduce and oxidize redoxactive molecules repeatedly and reversibly. Each analyte molecule thus contributes multiple electrons to the faradaic current, amplifying the detected signal. Redox cycling is naturally suited for use in nanofluidic systems since the degree of amplification increases with decreasing separation between the electrodes. Here, we introduce a device consisting of interdigitated electrodes imbedded in a nanofluidic channel that combines ease of fabrication with high levels of amplification and demonstrate its capabilities using paracetamol as a prototypical analyte.

\section{Redox cycling}

Amperometric reactions individually involve only one or a few electrons per reacting molecule, rendering the direct detection of single or small numbers of molecules in solution virtually impossible. This obstacle can be overcome by redox cycling, a technique in which each target molecule travels between electrodes repeatedly, reversibly changing its charge state with each electrode interaction. Thus, each molecule, over time, contributes hundreds or thousands of electrons to the measured current.

In conventional voltammetric techniques, a potential (most commonly varying linearly in time) is applied to a single working electrode to oxidize or reduce species present nearby in solution, and the current resulting from the redox process is measured as a function of potential. The resulting voltammogram provides information about the chemical identity of the redox species (position of the features on the potential axis) as well as the concentration (magnitude of the current at a given potential). The shape of the voltammogram can also be analyzed in further detail to yield information about the thermodynamics and kinetics of the electrode reaction.

Redox cycling requires at least two working electrodes. The electrodes are biased at sufficiently cathodic and anodic potentials relative to the formal potential of the given reaction that the product of the reduction at one electrode can diffuse to the other electrode where it undergoes oxidation and vice versa. This cycle is illustrated in Fig. 1.

For two planar electrodes separated by a thin layer of fluid, diffusion of redox molecules between the two

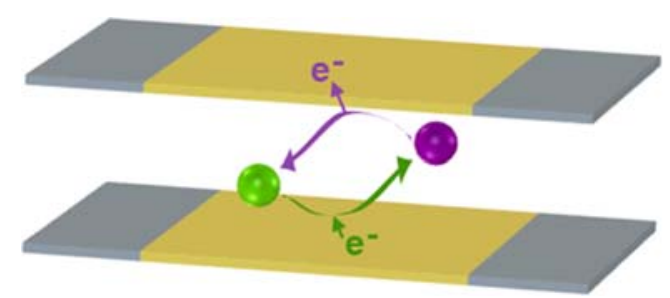

Fig. 1 Illustration of redox cycling. The molecule is oxidized at the top electrode, and after diffusing to the bottom, electrode is reversibly reduced back to its original state. Every cycle, one electron (or more, depending on the species) is shuttled by the molecule from one electrode to the other, giving rise to a steady-state electrochemical current

electrodes is essentially one dimensional. The corresponding steady-state current, $I$, is then given by

$I=\frac{n F S D C}{z}$.

Here, $n$ is the number of electrons transferred per molecule, $F$ is the Faraday constant, $S$ is the area of overlap between the two electrodes, $D$ is the diffusion constant of the redox species, $C$ is the bulk concentration of the redox species, and $z$ is the distance between the electrodes. This equation assumes that the transport of redox species is due solely to diffusion, a valid approximation as long as an excess of supporting electrolyte is present such that the Debye length is much smaller than $z$. Equation 1 also neglects any contribution from fringing of the diffusion profile around the edge of the electrode; this contribution to the current is negligible so long as $z$ is much smaller than the lateral dimensions of the electrodes. Equation 1 indicates that the degree of amplification of the amperometric signal increases as the electrode spacing, $z$, is made smaller. Efficient redox cycling thus requires a method for accurately controlling the distance between two electrodes.

In standard amperometric detection, each molecule gains or loses $n$ electrons only once. Therefore, a useful figure of merit for quantifying the effectiveness of redox cycling is the average number of times that a given molecule gains and loses $n$ electrons during a measurement. We define this value as the amplification factor, $A$. Since the amplification factor is independent of the concentration or the choice of redox species, it facilitates comparison of results obtained by different groups measuring under different conditions. It is easiest to measure in systems where the faradaic current reaches a steady state. The amplification factor is then simply the ratio of the redox cycling current flowing through the working electrodes inside the nanochannel, $I_{\mathrm{rc}}$, to the current $I_{\mathrm{ref}}$ simultaneously flowing through the reference electrode outside of the nanochannel: $A=I_{\mathrm{rc}} / I_{\mathrm{ref}}$. Since conventional electrochemistry instrumentation does not allow measurement of the current through the reference electrode, an alternative means of extracting $A$ is to measure the ratio of the current flow under redox cycling conditions 
versus the case where only one electrode has a potential applied while the other is left floating, $I_{1}$, and using $A=2 I_{\mathrm{rc}} /$ $I_{1} \cdot{ }^{1}$

The main limitation of redox cycling as a detection scheme is that it can only be applied to molecules that undergo reversible redox cycling. Also, as in conventional voltammetry, the potential window that can be used for detection is limited because of other unavoidable reactions, such as reduction and oxidation of water or solutes.

Interdigitated electrodes

Interdigitated electrodes (IDEs), also known as interdigitated arrays or band-array electrodes, are often employed in electrochemical detection. IDEs have been fabricated and analyzed since the 1980s [24-28]. They are primarily employed as conductometric [29-31] and amperometric sensors. As amperometric sensors, they are often used in the detection of biomolecules [32-34] and have even been employed as detectors in capillary electrophoresis $[8,35$, 36]. A sketch of an IDE is shown in Fig. 2. For amperometric detection, the sensitivity of IDEs is determined by how small the spacing between electrodes can be made, which is set by fabrication limits.

Recent fabrication improvements have rekindled interest in the technology. With electron-beam lithography becoming more widely available, there has been a move toward nanoscale electrode widths as well as spacing between electrodes. Several groups have demonstrated sub-micrometer wide electrodes and spacing [37-40], and novel geometries and designs have been analyzed using simulations [41-44].

A recently introduced approach is to purposely fabricate the electrodes out of plane by increasing the thickness of the electrode wires [37]. Dam et al. took this approach one step further and etched trenches in the substrate before patterning metal so that the electrodes face each other [45]. By reducing the space between electrodes, the chance that a molecule residing in the space between electrodes will undergo redox cycling increases. Using this technique, amplification factors $A$ in the range of 60-70 were achieved.

\section{Nanofluidic TLCs}

Microfabrication techniques also offer an opportunity to construct thin-layer cells (TLCs) in which the electrode spacing is below $100 \mathrm{~nm}[40,46,47]$. We refer to these here as nanofluidic TLCs (nF-TLCs). To achieve the smaller

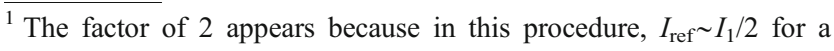
device with a symmetric IDE geometry. This is because $50 \%$ of the redox species is in the oxidized state, while the other $50 \%$ is in the reduced state in the region during the measurement of $I_{\text {ref }}$ while they are completely reduced or oxidized during the measurement of $I_{1}$.
}

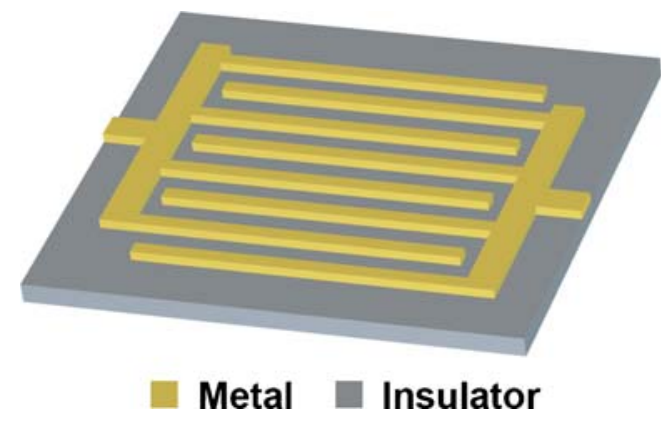

Fig. 2 Schematic diagram of an IDE viewed from above

spacing, the fabrication is based on a sacrificial layer instead of bulk micromachining [10]. The advantages of this approach are that small dimensions can be achieved in a controlled and reproducible manner, that the lateral geometry of the devices can be controlled at will, and that the devices can easily be integrated into micro-/nanofluidic systems. The devices are however challenging to fabricate in that great care must be taken to ensure that stress in the roof material is eliminated to prevent warping of the top electrode.

Figure 3a shows a fabrication process developed in our group for nF-TLCs. A finished device is pictured in Fig. $3 \mathrm{~b}$. The devices offer extraordinary sensitivity: amplification

a

1)

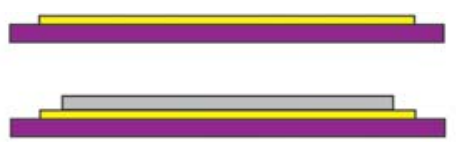

3)

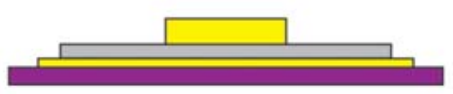

4)
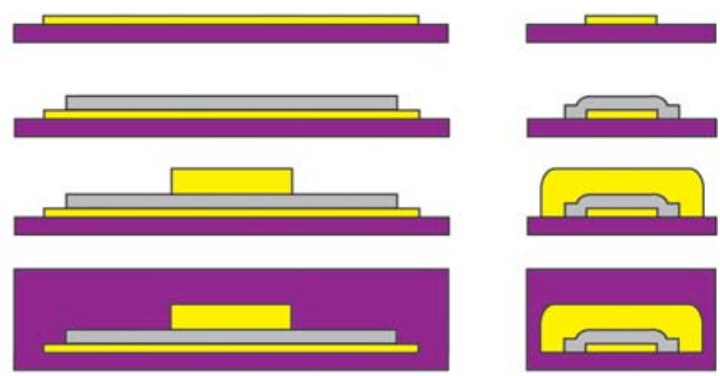

5)
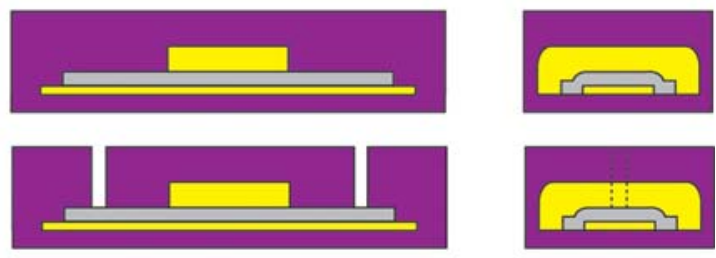

6)

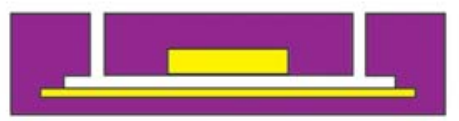

$\mathrm{SiO}_{2}$

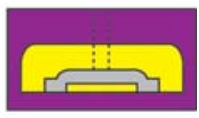

b

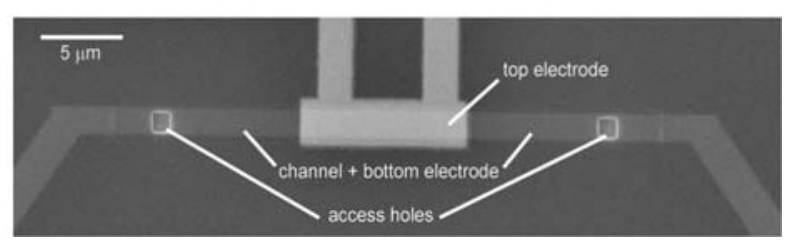

Fig. 3 a Fabrication schematic for nF-TLCs. 1 Pt is deposited and patterned on an oxidized silicon wafer. $2 \mathrm{~A} \mathrm{Cr}$ sacrificial layer is deposited. 3 A second layer of $\mathrm{Pt}$ is deposited and patterned to form the top electrode. $4 \mathrm{SiO}_{2}$ is deposited to enclose the device. 5 Access holes are defined in the $\mathrm{SiO}_{2} .6$ The $\mathrm{Cr}$ sacrificial layer is removed to create the nanochannel. b Scanning electron microscope image of a completed device 
factors as high as $\sim 10^{4}$ and detection limits approaching 100 molecules are possible for devices with channel heights of $25-55 \mathrm{~nm}[40,46,47]$.

\section{Selective amplification}

Redox cycling has the additional advantage that it offers some degree of selectivity to amperometric detection, which is achieved entirely through geometric constraints. In a mixture containing chemically reversible and irreversible species, the signal from species that can redox cycle is amplified by the amplification factor, $A$, while chemically irreversible processes only contribute one or a few electrons per molecule. This allows detection of species that undergo redox cycling in the presence of an excess concentration of chemically irreversible species, even when both species react at the same electrode potential. In order to maximize $A$, the residence time of molecules inside the detection volume has to be maximized, while the spacing between the electrodes, $z$, is minimized. Complete chemical irreversibility in the reactions of the interfering species is not required: so long as the lifetime of the reduced or oxidized form of this species is shorter than the residence time inside the device, some degree of selective enhancement is achieved.

Selective detection using this approach was observed in IDEs, but the molecules did not interact with the electrodes long enough to offer significant amplification of the target molecule [48]. The selectivity is significantly increased in out-of-plane IDEs; here, $A$ is larger since the molecules reside between the electrodes for extended amounts of time before diffusing back out to the bulk [45].

Wolfrum et al. recently demonstrated selective detection of catechol in the presence of ascorbic acid using nF-TLCs [46]. Since ascorbic acid decomposes quickly after being oxidized, it does not contribute significantly to the cycling current. Ascorbic acid was shown not to interfere with the detection of catechol up to 500:1 excess in this approach.

\section{Nanofluidic IDEs}

The nF-TLC devices discussed above provide enough amplification to detect a few hundred molecules, but the small volume of the active region of the device limits their molar sensitivity, which is more important in many applications. Increasing the volume by creating a large detection area $\left(>100 \mu \mathrm{m}^{2}\right)$ remains quite challenging using the fabrication scheme for nF-TLCs presented in the previous section due to the higher probability of defects in the sacrificial layer that lead to short circuits in the device. Here, we combine the simpler fabrication scheme offered by IDEs with the higher redox cycling amplification factors offered by nF-TLC geometries. The result is a hybrid device that we call a nanofluidic IDE (nF-IDE). A nF-IDE is an IDE device that has an upper wall or roof placed over the top of the electrodes to partially restrict the fluid volume in the vicinity of the detection area. This geometry differs from nF-TLCs in that the fluid is not completely confined in the cleft between the electrodes, but still offers a high degree of localization without changing the electrode height or spacing. Since the electrodes are patterned in the same plane using a single processing step, the electrode spacing is defined by the lithographic pattern. The $\mathrm{Cr}$ sacrificial layer is thus not involved in creating the separation between electrodes. Pattern definition using electron-beam lithography has nearly the same resolution limit as the thickness of the sacrificial layers employed to date in nF-TLCs. Figure 4 shows a schematic diagram of the device, with a nanochannel of height $h$ and spacing between electrodes $z$.

\section{Theory}

To illustrate the effect of creating a roof over an IDE, we numerically solved the diffusion equation to determine the maximum, diffusion-limited current as a function of device geometry. The calculations were performed for a simple two-dimensional model of our device geometry using finite element simulation software (Comsol 3.4, Stockholm, Sweden). Constant-concentration boundary conditions were applied at the electrode surfaces, which corresponded to applying high overpotentials to the electrodes so that complete oxidation or complete reduction takes place there. Figure 5a shows the computed concentration profiles for two devices with different roof heights $h=75$ and $h=200 \mathrm{~nm}$, where $h$ is defined in the figure. Both devices have 50-nmtall electrodes, spaced $z=250 \mathrm{~nm}$ apart. Figure $5 \mathrm{~b}$ shows the corresponding amperometric current for a $100 \mu \mathrm{M}$ concentration of a species with a diffusion coefficient $D=2.33 \times 10^{-10} \mathrm{~m}^{2} / \mathrm{s}$ and $n=2$ electrons transferred.

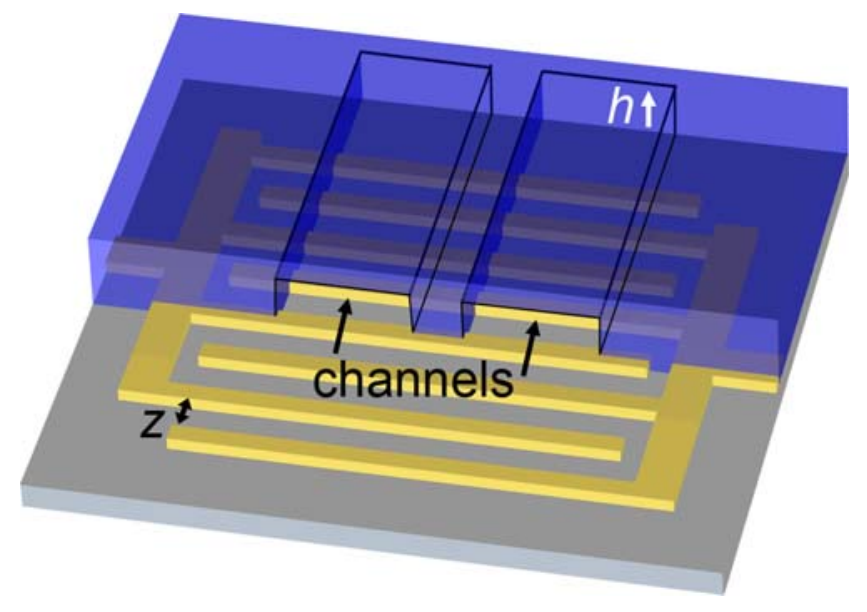

Fig. 4 Schematic diagram of a nF-IDE 
(a)
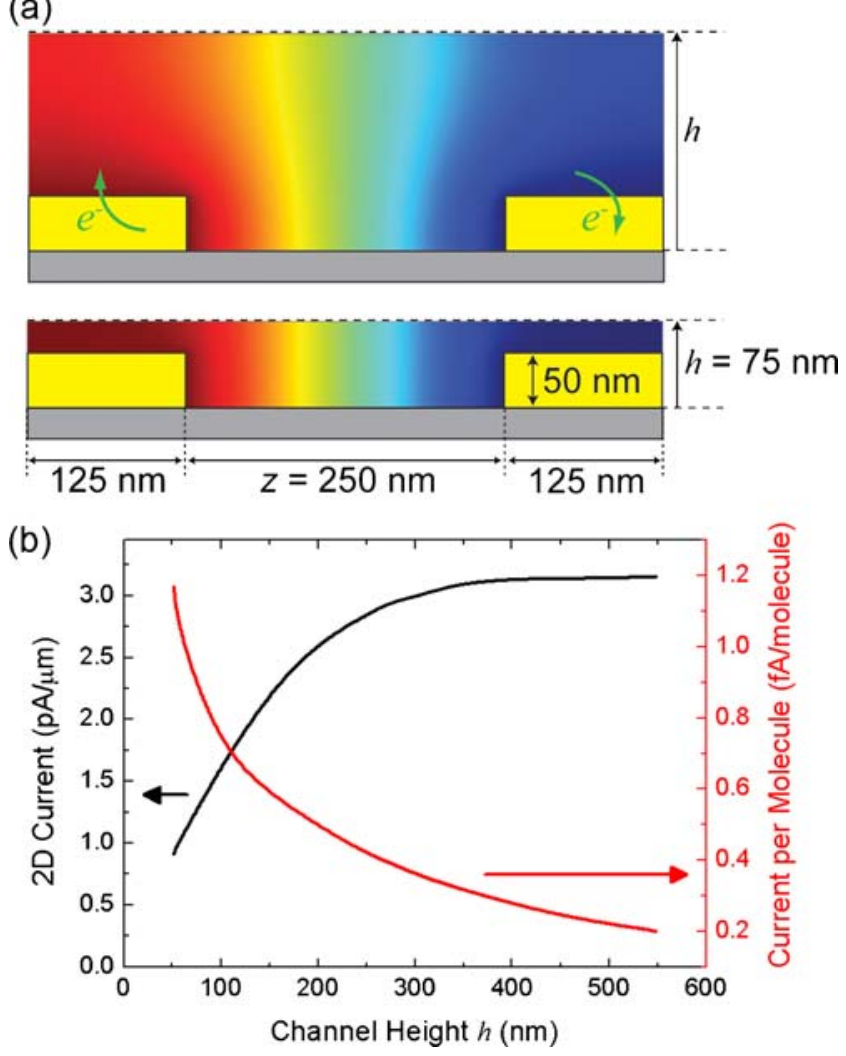

Fig. 5 a Concentration profiles in a nF-IDEs as determined by solving the two-dimensional diffusion equation. The color scale shows the concentration distribution of oxidized molecules in the nanochannel (red fully reduced, blue fully oxidized). The yellow rectangles represent the electrodes. b The black curve shows the current generated per unit length of electrode as a function of the height of the nanofluidic channel placed over the IDE with 50-nm-tall electrodes spaced $250 \mathrm{~nm}$ apart. The red curve shows the current per molecule for the same device. These results are for a $100 \mu \mathrm{M}$ concentration of a molecule (paracetamol) that transfers two electrons per cycle and has a diffusion coefficient of $2.33 \times 10^{-10} \mathrm{~m}^{2} / \mathrm{s}$

These parameters correspond to paracetamol, which was used to test the nF-IDE devices. $D$ was determined from the steady-state current at a conventional Pt disk ultra-micro electrode (UME, BASi, Stareton, United Kingdom). The linear current density can be converted into a total current by multiplying by the length of the electrodes and by the number of electrode fingers in a given device.

Figure $5 \mathrm{~b}$ indicates that the magnitude of the current (black curve) increases with increasing roof height, $h$ matches the spacing between the IDEs, at which point it saturates to a value that corresponds to a conventional IDE with no roof. The increase of the current is due to the fact that the number of molecules present in the cavity for a given concentration increases with $h$. Even as the total current increases, however, the current contributed by each molecule in the channel decreases precipitously, as shown by the red curve in Fig. 5b. For $h$ much larger than the electrode spacing, the increase in current with $h$ due to the increasing number of molecules is counteracted by the reduction in the current per molecule. A large current per molecule corresponds to efficient redox cycling and is achieved with small roof heights. In the limit $h \rightarrow 50 \mathrm{~nm}$, the device resembles a TLC, and the current per molecule is simply $n e D / z^{2}(=1.2 \mathrm{fA}$ for $n=2$ and $z=250 \mathrm{~nm})$, where $e$ is the charge of one electron [18, 40]. Controlling the height of the roof, $h$, therefore allows tuning between regimes of efficient cycling and high selectivity $(h \rightarrow 50$ $\mathrm{nm})$ and maximum signal level $(h \rightarrow \infty)$.

\section{Experimental section}

Device fabrication

Devices were fabricated using standard lithographic techniques. Figure 6 shows a sketch of the fabrication scheme. Ideally, a single broad nanochannel would be constructed over the entire array of IDEs, but the roof material that we employ exhibits significant intrinsic strain. This would cause significant deformation of the roof and could cause the resulting nanochannel height to deviate significantly from its designed value upon removing the sacrificial layer. To minimize this effect, we designed a series of narrow nanochannels that are placed over the IDE device. While a fraction of the IDE surface is then unused, the walls between the nanochannels provide added support and greatly reduce deviations in channel height. Figure $7 \mathrm{a}$ shows an optical image of a nF-IDE device with the $\mathrm{Cr}$ sacrificial layer still in place. The nanochannel layout used in this paper employs 24 nanochannels, $75 \mathrm{~nm}$ tall, $3 \mu \mathrm{m}$ in width, with a $1 \mu \mathrm{m}$ wall between channels. Figure $7 \mathrm{~b}$ is a zoomed-in image of the lower left corner of the device; 250-nm-wide IDEs with $250 \mathrm{~nm}$ spacing are visible at the top of the image. The length of the IDE array is $100 \mu \mathrm{m}$.

The nF-IDEs do not have a planar nanochannel roof. Since the electrodes have a thickness of $50 \mathrm{~nm}$ and the $\mathrm{Cr}$ sacrificial layer has a uniform $75 \mathrm{~nm}$ thickness, the roof instead has a corrugated shape reflecting the topography of the electrodes. Simulations show that this deviation from the idealized geometry of Fig. 5 has a negligible impact on the magnitude of the current.

A polished and diced 4-in. silicon wafer with $500 \mathrm{~nm}$ of thermally grown $\mathrm{SiO}_{2}$ was used as the substrate. The wafers were cleaned in acetone and isopropylalcohol (IPA) prior to use. First, the wafer was spin-coated with polymethylmethacrylate (PMMA) 950K (4\% in anisole) at 2,500 rpm for $60 \mathrm{~s}$, followed by baking at $175{ }^{\circ} \mathrm{C}$ for $5 \mathrm{~min}$. The PMMA was exposed in a Leica (EBPG 5000) electron-beam pattern generator using a dose of $1,000 \mu \mathrm{C} / \mathrm{cm}^{2}$ (high tension $100 \mathrm{kV}$, aperture $400 \mu \mathrm{m}$, current $159 \mathrm{nA}$, BSS $70 \mathrm{~nm}$, spot size $89 \mathrm{~nm}$ ). The exposed parts were developed using a 1:3 
Fig. 6 Fabrication process for nF-IDEs. a Pt is deposited and patterned to create the IDEs. b A $\mathrm{Cr}$ sacrificial layer is deposited and patterned. c $\mathrm{SiO}_{2}$ is deposited to enclose the structure and patterned to create access holes. d The $\mathrm{Cr}$ layer is removed to create nanochannels on top of the IDEs
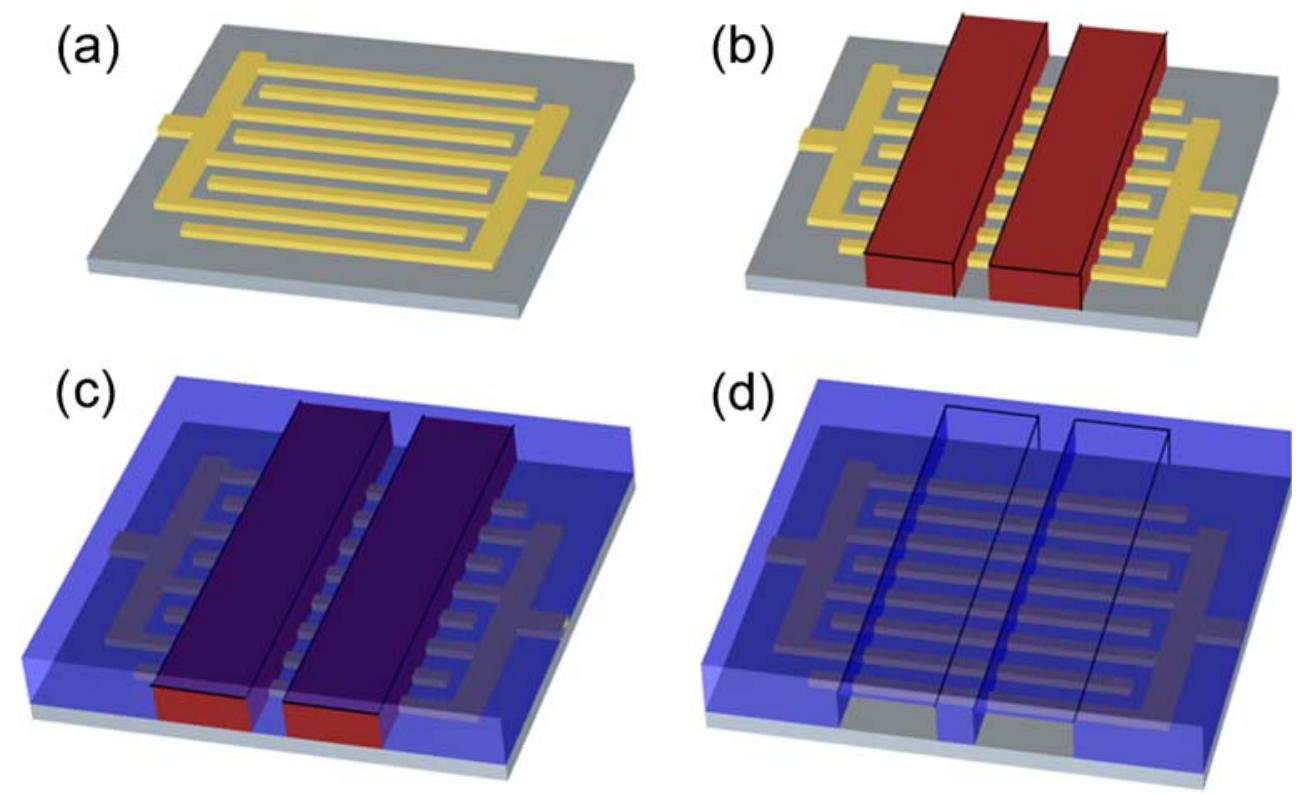

mixture of methylisobutylketone/IPA for $70 \mathrm{~s}$, followed by a 30-s rinse in IPA.

After development, the wafer was coated with $4 \mathrm{~nm} \mathrm{Ti}$ (0.5 $\AA / \mathrm{s})$ as an adhesive layer using electron-beam evaporation, followed by $50 \mathrm{~nm}$ Pt $(0.7 \AA / \mathrm{s})$ and $2 \mathrm{~nm}$ of $\mathrm{Cr}(0.3 \AA / \mathrm{s})$ as the top adhesion layer. Metal lift-off was carried out by placing the wafer in boiling acetone to remove the unexposed PMMA. A new layer of PMMA $950 \mathrm{~K}$ ( $9 \%$ in anisole) was then spun on the device at $2,500 \mathrm{rpm}$ and baked for $5 \mathrm{~min}$ at $175^{\circ} \mathrm{C}$. The PMMA was exposed and developed using the same process as in the previous step, but with a different pattern that defines the nanochannels over the IDE. The nanochannels were created by depositing $75 \mathrm{~nm}$ of $\mathrm{Cr}(0.6 \AA / \mathrm{s})$ and performing metal lift-off. The channels were enclosed by sputter deposition of $245 \mathrm{~nm}$ of $\mathrm{SiO}_{2}$. Access holes were defined by patterning PMMA as before. The holes were created using reactive ion etching (Leybold, Cologne, Germany; $\mathrm{O}_{2}$ $\left.2.0 \mathrm{sccm}, \mathrm{CHF}_{3} 50 \mathrm{sccm}, 50 \mu \mathrm{bar}, 90 \mathrm{~W}\right)$. The individual devices were diced from the wafer and mounted into polydimethylsiloxane (PDMS) microfluidic flow cells. The sacrificial $\mathrm{Cr}$ layer was etched away just prior to using the devices using Cr etchant [49].

\section{Reagents}

Paracetamol and potassium chloride were obtained from Sigma-Aldrich (the Netherlands). Chromium etchant and ascorbic acid were purchased from Merck (the Netherlands). All chemicals were used without further purification. The solutions were passed through an Anotop 20-nm filter (Whatman, Madistone, England) before use. All solutions were prepared in $1.5 \mathrm{M} \mathrm{KCl}$ and $0.5 \mathrm{M}$ sodium phosphate buffer (PB) with Millipore water (Milli-Q) with a final pH of 6.3.
Measurements

Cyclic voltammograms were recorded with a bipotentiostat (CHI832B, CH Instruments). A standard $\mathrm{Ag} / \mathrm{AgCl}$ electrode (3M NaCl, BASi, Stareton, United Kingdom) that served as both a reference and counter electrode was immersed in a PDMS flow cell that contacted the device. Due to the low currents flowing through the reference electrode in our measurements $(<1 \mathrm{nA})$, a separate counter electrode was not necessary. The two interdigitated electrodes served as working electrodes. Voltammograms were obtained by cycling the potential at one electrode while the potential at the other was held constant at $0 \mathrm{~V}$.

A flow cell was connected to the device to provide a fluidic interface and to integrate the reference electrode in the setup. After use, the chips were cleaned in Milli-Q water, dried with nitrogen gas, and stored for future use. Devices could be reused multiple times. All of the experiments were conducted in a Faraday cage to reduce electromagnetic interference.

\section{Results and discussion}

For these proof-of-concept experiments using nF-IDEs, we used paracetamol as the redox-active species. Paracetamol is a common ingredient in many pain medications [50]. Because overdoses can be lethal, testing of urine for paracetamol is a common procedure [51]. Its presence in urine samples can also interfere with tests for other catechols [52]. Paracetamol thus represents an ideal prototypical system for exploring the capabilities of new sensor devices.

Figure 8 compares voltammograms obtained in one- and two-electrode modes for solutions containing $200 \mu \mathrm{M}$ 

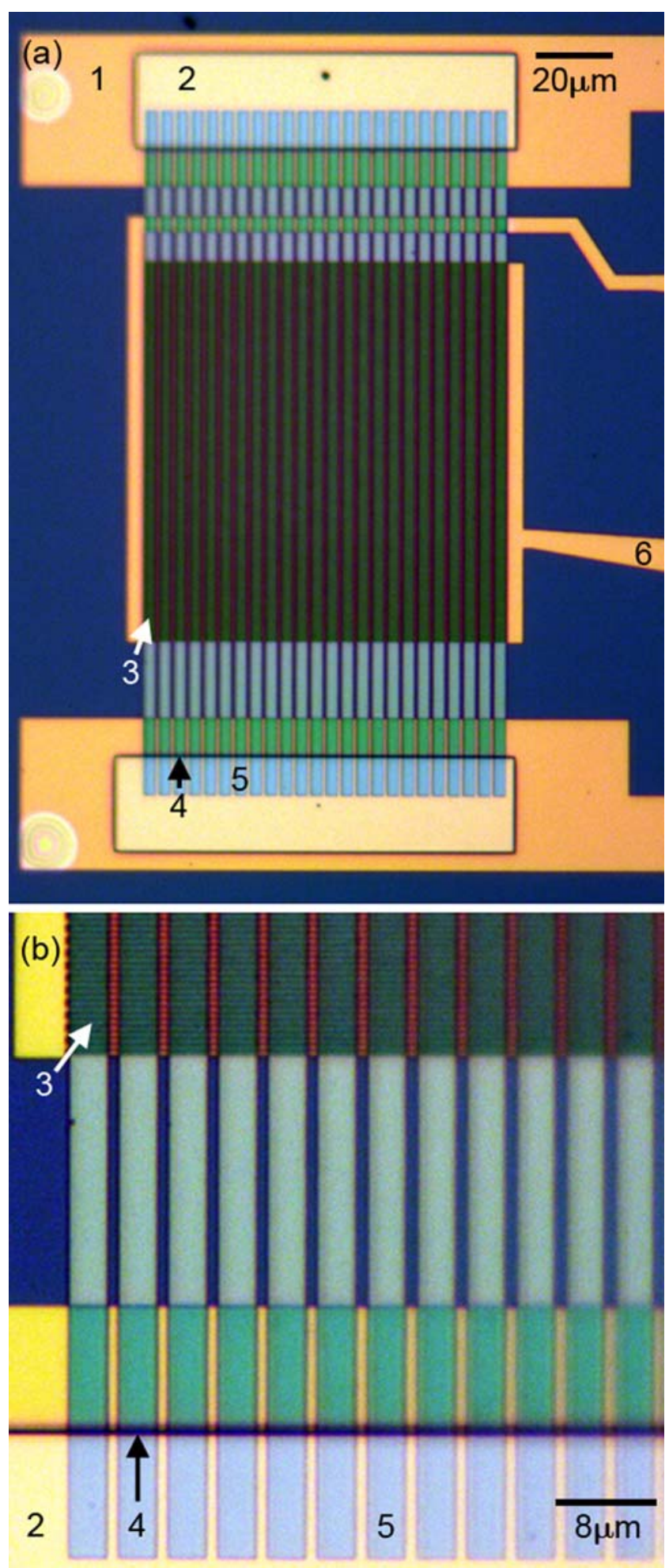

Fig. 7 a Micrograph image of a nanofluidic IDE. The Cr sacrificial layer has not yet been removed in this device, corresponding to the structure in Fig. 6c. The device has 24 nanochannels, each $3 \mu \mathrm{m}$ wide, constructed using a 75-nm-thick $\mathrm{Cr}$ sacrificial layer, patterned over 250-nm-wide IDEs with $250 \mathrm{~nm}$ spacing. b Zoomed-in image of the lower left-hand part of the device. 1 Preconditioning electrodes, not used; 2 access holes connecting bulk solution to nanochannels; 3 IDEs; 4 nanofluidic channels; $5 \mathrm{Cr}$ sacrificial layer; 6 wires to external connections

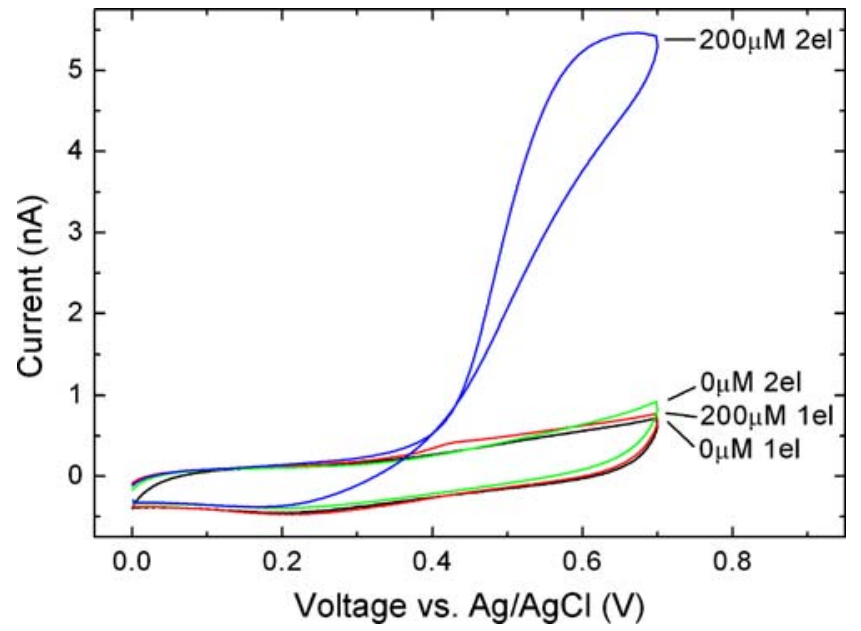

Fig. 8 Signal amplification due to redox cycling (black one electrode, buffer only; red one electrode, $200 \mu \mathrm{M}$ PC; green redox cycling, buffer only; blue redox cycling, $200 \mu \mathrm{M} \mathrm{PC}$ ). In one-electrode mode, the second electrode is kept floating while the other is swept. In redox cycling mode, the second electrode is maintained at a constant potential of $0 \mathrm{~V}$. A scan rate of $25 \mathrm{mV} / \mathrm{s}$ was used for both detection modes. An amplification factor of 110 is achieved using nF-TLCs with $250 \mathrm{~nm}$ IDEs and nanochannels made with a 75-nm-thick $\mathrm{Cr}$ sacrificial layer

paracetamol or pure buffer only. A clear wave is observed in redox cycling mode, whereas the corresponding wave in one-electrode mode is well-defined but small compared to the background signal. The redox cycling voltammogram also exhibits hysteresis, suggesting that potential-dependent adsorption is occurring [46]. These results correspond to an amplification factor of $\sim 110$, illustrating the effectiveness of redox cycling amplification inside of a nanochannel device. This value is larger than the highest amplification values reported for uncovered IDEs $(A \approx 70)$ [45] but, as expected, is drastically lower than nF-TLCs, which confine the sample within a $55 \mathrm{~nm}$ channel $\left(A \approx 10^{4}\right)[40,46,47]$. Next, we compared the maximum current achieved in our devices to the finite element model. The predicted linear current density from finite element calculations for $h=$ $75 \mathrm{~nm}$ and $200 \mu \mathrm{M}$ paracetamol concentration is $2.53 \mathrm{pA} /$ $\mu \mathrm{m}$, which corresponds to a total diffusion-limited current of $36.5 \mathrm{nA}$. The current measured in our devices is therefore about seven times lower than predicted. While this is a significant deviation, it is not unexpected. The diffusion-limited current can only be obtained when reaction kinetics at the electrode surface are not influencing the current. The fact that the voltammograms do not exhibit an ideal sigmoidal shape with a plateau at high potential indicates that such kinetic limitations are present. Potential-dependent adsorption to the electrode surfaces may also play a role; such non-ideal voltammograms have also been observed in nF-TLCs [46]. We also tested devices with $h=120 \mathrm{~nm}$. The amplification factor, $A$, for these devices was approximately 30 , which was considerably 
lower than for devices with $h=75 \mathrm{~nm}$, as expected from modeling.

In order to test the response and determine the detection limit of our devices, we obtained cyclic voltammograms using a range of concentrations of paracetamol. Figure 9a shows cyclic voltammograms for paracetamol concentrations ranging from $1 \mu \mathrm{M}$ to $1 \mathrm{mM}$ using a scan rate of $25 \mathrm{mV} \mathrm{s}^{-1}$. Next, the maximum cycling currents from the voltammograms were plotted for each concentration (Fig. 9b). The response of the devices is linear for concentrations ranging from $10 \mu \mathrm{M}$ to $1 \mathrm{mM}$. The lower detection limit with these scan parameters is approximately $10 \mu \mathrm{M}$, while the upper detection limit is near the solubility limit of paracetamol. In the future, the lower detection limit can be reduced further by decreasing the spacing between electrodes.

We have also tested the selectivity of our devices by detecting paracetamol in the presence of ascorbic acid. Since paracetamol testing is usually carried out with complex samples, it is important to be able to detect the
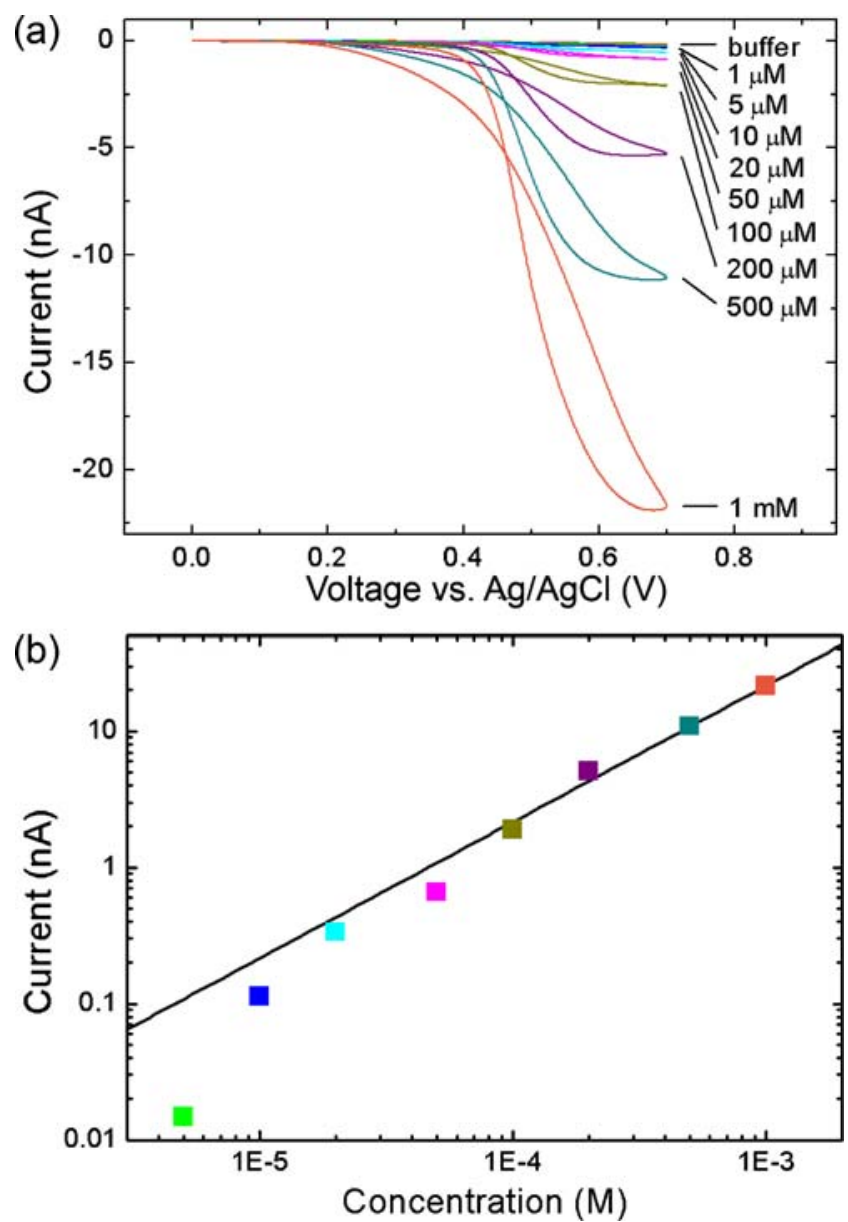

Fig. 9 Detection limit of paracetamol using nF-IDE. a Cyclic voltammograms of the reductive currents obtained for paracetamol concentrations ranging from $1 \mu \mathrm{M}$ to $1 \mathrm{mM}$ (black buffer; red $1 \mu \mathrm{M}$; green $5 \mu \mathrm{M}$; blue $10 \mu \mathrm{M}$; cyan $20 \mu \mathrm{M}$; magenta $50 \mu \mathrm{M}$; olive $100 \mu \mathrm{M}$; purple $200 \mu \mathrm{M}$; turquoise $500 \mu \mathrm{M}$; orange $1 \mathrm{mM}$ ). b Plot of the maximum wave height obtained from a versus concentration paracetamol signal in the presence of interfering molecules $[53,54]$. Figure 10 a shows cyclic voltammograms obtained by measuring solutions with $100 \mu \mathrm{M}$ paracetamol and varying concentrations of ascorbic acid using a $10-\mu \mathrm{m}-$ diameter Pt UME (BASi, Stareton, United Kingdom). An UME was used for comparison in this case because the $\mathrm{nF}$ IDE gives no measureable signal in one-electrode mode for these concentrations. In this case, no redox cycling can occur. The current generated by the ascorbic acid contributes significantly to the total signal, thereby masking the current originating from the paracetamol. The deviation from ideal mass transport limited cyclic voltammograms in Fig. 10 is most likely caused by sluggish kinetics for the molecules and potential-dependent adsorption of species to the electrode. Similar behavior was observed in nF-TLCs [46].
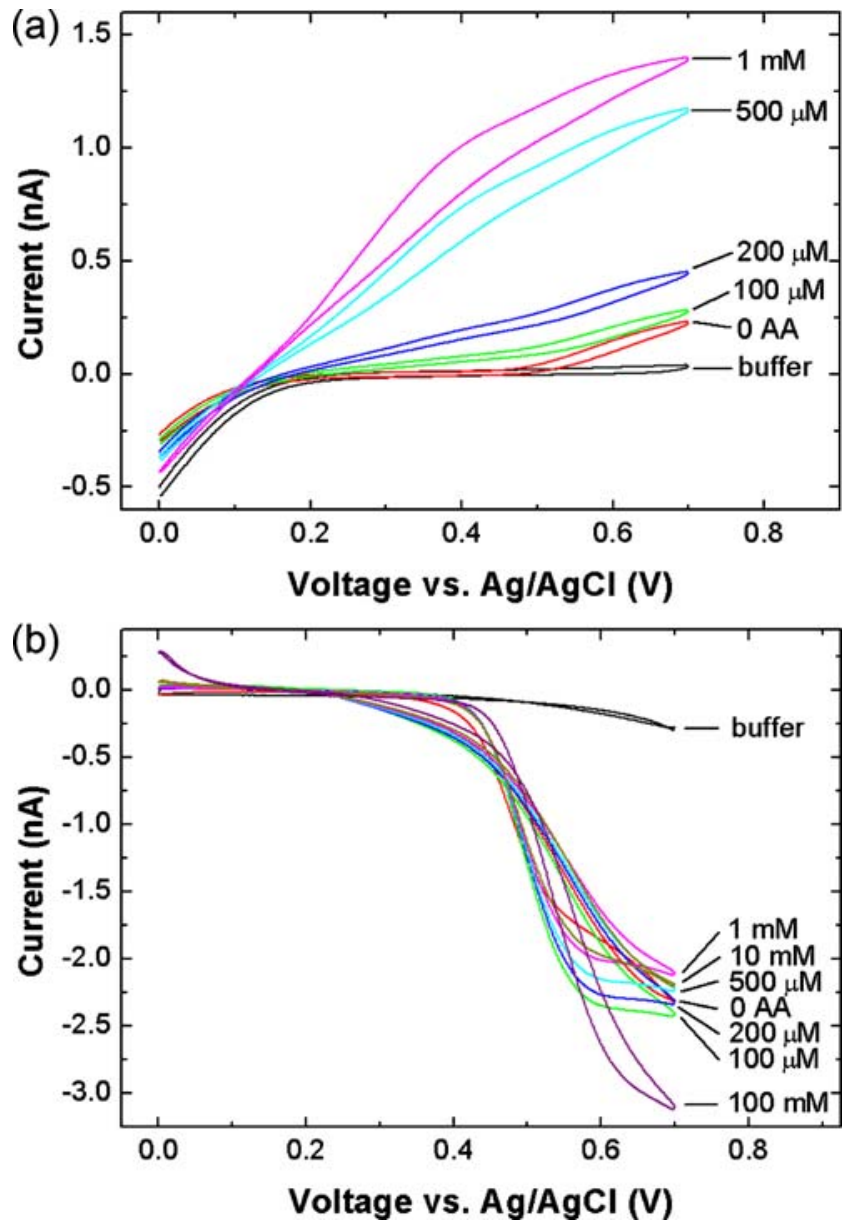

Fig. 10 a Cyclic voltammograms for $100 \mu \mathrm{M}$ paracetamol and various concentrations of ascorbic acid in $1.5 \mathrm{M} \mathrm{KCl}+0.5 \mathrm{M}$ phosphate buffer ( $\mathrm{pH}$ 6.3) using a 10- $\mu \mathrm{m}$-diameter UME (scan rate $25 \mathrm{mV} / \mathrm{s}$ ). The ascorbic acid signal overlaps and masks the signal generated by paracetamol. b Cyclic voltammograms for $100 \mu \mathrm{M}$ paracetamol using a nF-IDE device in $1.5 \mathrm{M} \mathrm{KCl}+0.5 \mathrm{M} \mathrm{PB}$ and various concentrations of ascorbic acid. One electrode was swept at $25 \mathrm{mV} / \mathrm{s}$, while the other electrode was kept at a potential of $0 \mathrm{mV}$ vs. $\mathrm{Ag} / \mathrm{AgCl}$ (black buffer; red $0 \mathrm{AA}$; green $100 \mu \mathrm{M}$; blue $200 \mu \mathrm{M}$; cyan $500 \mu \mathrm{M}$; magenta $1 \mathrm{mM}$; olive $10 \mathrm{mM}$; purple $100 \mathrm{mM}$ ) 

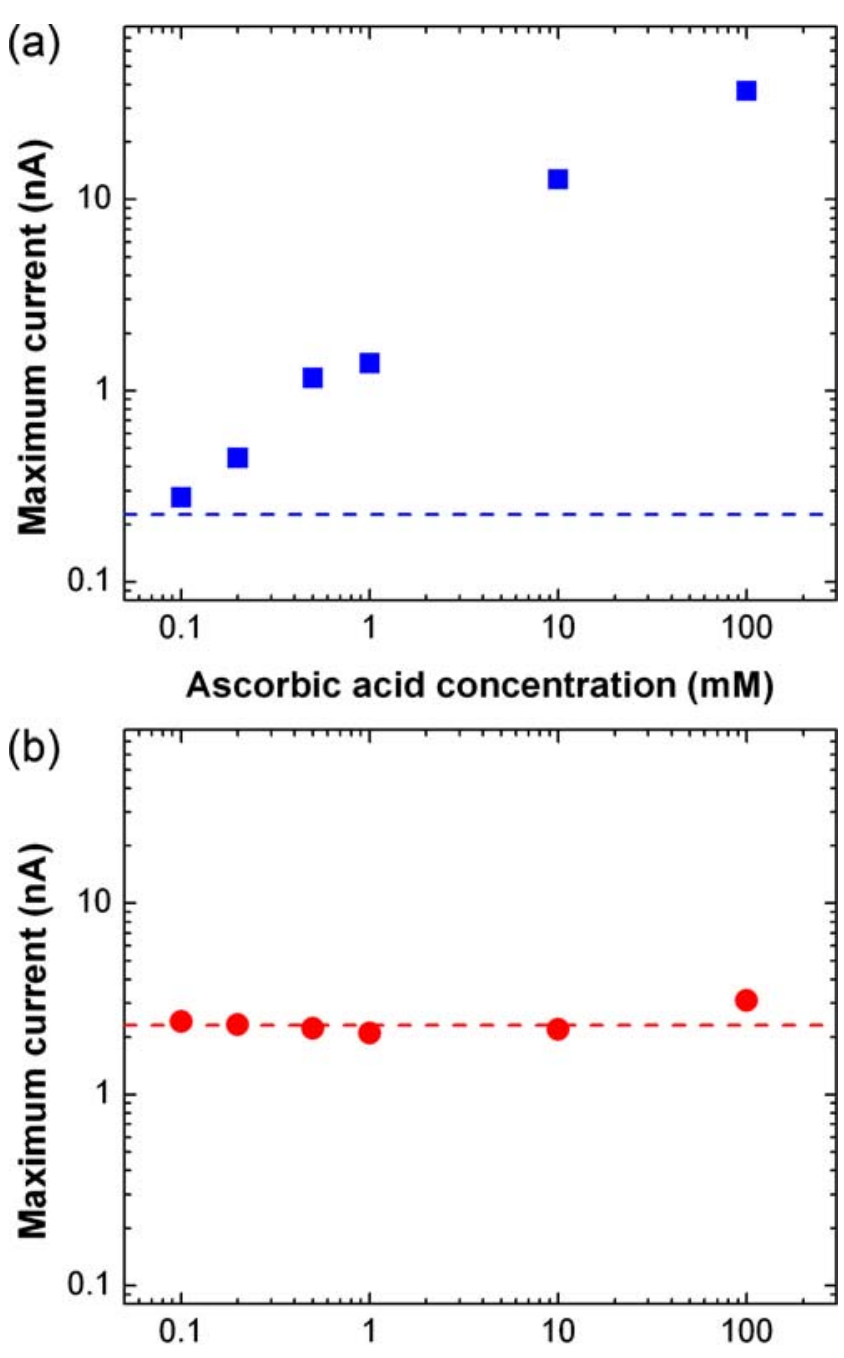

Ascorbic acid concentration ( $\mathrm{mM}$ )

Fig. 11 Plot of the peak currents for $100 \mu \mathrm{M}$ paracetamol in the presence of varying ascorbic acid concentrations measured using a a 10 - $\mu \mathrm{m}$-diameter UME (blue squares) and $\mathbf{b}$ a $\mathrm{nF}$-IDE device in redox cycling mode (red circles). Blue and red dashed lines represent the current obtained with $100 \mu \mathrm{M}$ paracetamol and no ascorbic acid added for UME and nF-IDE devices, respectively. For the nF-IDE, only the current from the reducing electrode is shown

Since the oxidation product of ascorbic acid is only stable on the order of milliseconds [55, 56], its influence on the amplified redox cycling current is expected to decay quickly inside the nanochannel. Figure 10b shows cyclic voltammograms from the reducing electrode obtained with a nF-IDE filled with $100 \mu \mathrm{M}$ paracetamol and varying concentrations of ascorbic acid. The maximum signals obtained from the UME and nF-IDE devices at various ascorbic acid concentrations are compared in Fig. 11. The relative contribution of ascorbic acid to the total signal is suppressed when redox cycling is enabled. For high enough concentrations $(>10 \mathrm{mM})$, however, we begin to see a current contributed by the ascorbic acid. The device can therefore accurately detect paracetamol for ascorbic acid present up to a 100:1 excess.

\section{Conclusions}

We have developed a redox sensor based on interdigitated electrodes imbedded inside a nanofluidic channel. These nanofluidic IDEs offer a simpler and more robust fabrication scheme than nF-TLCs as well as larger detection volumes. The amplification factor, while lower than in $\mathrm{nF}$ TLCs, can be tuned by adjusting the roof height. Tailoring the device geometry thus allows a tradeoff between maximal molar sensitivity and selectivity.

We fabricated $\mathrm{nF}$-IDEs with 250-nm-wide interdigitated electrodes spaced $250 \mathrm{~nm}$ apart that were covered with nanofluidic channels created by using a 75 -nm-thick $\mathrm{Cr}$ sacrificial layer. The devices were tested using paracetamol. An amplification factor of 110 was obtained, and a lower detection limit of $10 \mu \mathrm{M}$ was established. Further reduction of the electrode spacing promises additional enhancement in sensitivity. We have further shown that the technique is capable of detecting paracetamol without interference from ascorbic acid over a wide range of ascorbic acid concentrations.

Amperometric detection and nanofluidics complement each other well to provide a relatively easy to implement, label-free detection strategy. In particular, nanofluidic approaches can enhance conventional amperometric assays by improving sensitivity and selectivity. Nanofluidic devices can also be manufactured using standard microelectronics fabrication techniques and can thus be mass-produced. Once fabricated, the devices can be stored indefinitely and used as needed. We have tested devices after up to 1 year of storage prior to $\mathrm{Cr}$ etching and have not observed any change in their characteristics.

Acknowledgments This work was funded by the NanoNed and NWO. Edgar Goluch would like to thank the U.S. National Science Foundation for support through IRFP Grant Number 0754396. Bernhard Wolfrum was funded by the DFG. We acknowledge Cees Dekker for general support and helpful discussions.

\section{References}

1. Chen ZL, Hayashi K, Iwasaki Y, Kurita R, Niwa O, Sunaawa K (2005) Electroanal 17:231-238

2. Lisdat F, Wollenberger U, Makower A, Hortnagl H, Pfeiffer D, Scheller FW (1997) Biosens Bioelectron 12:1199-1211

3. Ciszewski A, Milczarek G (1999) Anal Chem 71:1055-1061

4. Malem F, Mandler D (1993) Anal Chem 65:37-41

5. Raoof JB, Ojani R, Rashid-Nadimi S (2005) Electrochim Acta 50:4694-4698

6. Selvaraju T, Ramaraj R (2003) Electrochem Commun 5:667-672 
7. Cvacka J, Quaiserova V, Park J, Show Y, Muck A, Swain GM (2003) Anal Chem 75:2678-2687

8. Martin RS, Gawron AJ, Lunte SM, Henry CS (2000) Anal Chem 72:3196-3202

9. Shin DC, Sarada BV, Tryk DA, Fujishima A (2003) Anal Chem 75:530-534

10. Abgrall P, Nguyen NT (2008) Anal Chem 80:2326-2341

11. Massari AM, Gurney RW, Schwartz CP, Nguyen ST, Hupp JT (2004) Langmuir 20:4422-4429

12. Krapf D, Quinn BM, Wu MY, Zandbergen HW, Dekker C, Lemay SG (2006) Nano Lett 6:2531-2535

13. Schoch RB, Cheow LF, Han J (2007) Nano Lett 7:3895-3900

14. Garcia AL, Ista LK, Petsev DN, O'Brien MJ, Bisong P, Mammoli AA, Brueck SRJ, Lopez GP (2005) Lab Chip 5:1271-1276

15. White RJ, White HS (2008) Langmuir 24:2850-2855

16. Sun P, Mirkin MV (2008) J Am Chem Soc 130:8241-8250

17. Georganopoulou DG, Mirkin MV, Murray RW (2004) Nano Lett 4:1763-1767

18. Fan FRF, Bard AJ (1995) Science 267:871-874

19. Brown FO, Lowry JP (2003) Analyst 128:700-705

20. Gonon F, Buda M, Cespuglio R, Jouvet M, Pujol JF (1980) Nature 286:902-904

21. Heien M, Khan AS, Ariansen JL, Cheer JF, Phillips PEM, Wassum KM, Wightman RM (2005) Proc Natl Acad Sci U S A 102:10023-10028

22. Rice ME, Oke AF, Bradberry CW, Adams RN (1985) Brain Res 340:151-155

23. Stamford JA (1985) Brain Res Rev 10:119-135

24. Rubinstein I (1995) Physical electrochemistry: principles, methods, and applications. Marcel Dekker, New York

25. Montenegro MI, Queiros MA, Daschbach JL, eds. (1991) Microelectrodes: theory and Applications. Kluwer, Dordrecht, the Netherlands

26. Sanderson DG, Anderson LB (1985) Anal Chem 57:2388-2393

27. Chidsey CE, Feldman BJ, Lundgren C, Murray RW (1986) Anal Chem 58:601-607

28. Niwa O, Morita M, Tabei H (1990) Anal Chem 62:447-452

29. Sheppard NF, Tucker RC, Wu C (1993) Anal Chem 65:1199-1202

30. Timmer B, Sparreboom W, Olthuis W, Bergveld P, van den Berg A (2002) Lab Chip 2:121-124

31. Jaffrezic-Renault N, Dzyadevych SV (2008) Sensors 8:25692588
32. Niwa O, Morita M, Tabei H (1991) Electroanal 3:163-168

33. Vandaveer WR IV, Woodward DJ, Fritsch I (2003) Electrochim Acta 48:3341-3348

34. Nebling E, Grunwald T, Albers J, Schafer P, Hintsche R (2004) Anal Chem 76:689-696

35. Male KB, Luong JHT (2003) J Chromatogr A 1003:167-178

36. Liu Z, Niwa O, Kurita R, Horiuchi T (2000) Anal Chem 72:13151321

37. Ueno K, Hayashida M, Ye J-Y, Misawa H (2005) Electrochem Commun 7:161-165

38. Hayashi K, Takahashi J, Horiuchi T, Iwasaki Y, Haga T (2008) J Electrochem Soc 155:J240-J243

39. Bange A, Tu J, Zhu XS, Ahn C, Halsall HB, Heineman WR (2007) Electroanal 19:2202-2207

40. Zevenbergen MAG, Krapf D, Zuiddam MR, Lemay SG (2007) Nano Lett 7:384-388

41. Strutwolf J, Williams DE (2005) Electroanal 17:169-177

42. Yang XL, Zhang GG (2007) Sens Actuat B-Chem 126:624631

43. Min JH, Baeumner AJ (2004) Electroanal 16:724-729

44. Odijk M, Olthuis W, Dam VAT, van den Berg A (2008) Electroanal 20:463-468

45. Dam VAT, Olthuis W, Berg AVD (2007) Analyst 132:365-370

46. Wolfrum B, Zevenbergen M, Lemay S (2008) Anal Chem 80:972-977

47. Zevenbergen MAG, Wolfrum B, Goluch ED, Lemay SG. Unpublished results

48. Aoki A, Matsue T, Uchida I (1990) Anal Chem 62:2206-2210

49. Sparreboom W, Eijkel JCT, Bomer J, Berg AVD (2008) Lab Chip 8:402-407

50. Sotomayor MDPT, Sigoli A, Lanza MRV, Tanaka AA, Kubota LT (2008) J Brazil Chem Soc 19:734-743

51. Whelpton R, Fernandes K, Wilkinson KA, Goldhill DR (1993) Biomed Chromatogr 7:90-93

52. Davidson FD (2004) Ann Clin Biochem 41:316-320

53. Hayashi K, Iwasaki Y, Kurita R, Sunagawa K, Niwa O (2003) Electrochem Commun 5:1037-1042

54. Paixao T, Richter EM, Brito-Neto JGA, Bertotti M (2006) Electrochem Commun 8:9-14

55. Perone SP, Kretlow WJ (1966) Anal Chem 38:1760-1763

56. Wehmeyer KR, Wightman RM (1985) Anal Chem 57:19891993 